\title{
Geospatial web services and applications for infectious disease surveillance
}

\author{
S. Gao ${ }^{1}$, E. Oldfield ${ }^{2}$, D. Mioc ${ }^{1}$, X. Yi ${ }^{3}$ \& F. Anton ${ }^{4}$ \\ ${ }^{I}$ Department of Geodesy and Geomatics Engineering, \\ University of New Brunswick, Canada \\ ${ }^{2}$ New Brunswick Lung Association, Canada \\ ${ }^{3}$ Service New Brunswick, Canada \\ ${ }^{4}$ Department of Informatics and Mathematical Modeling, \\ Technical University of Denmark, Denmark
}

\begin{abstract}
Infectious diseases have the ability to expand their geographic range, jump species, become resistant to antimicrobial agents, and have become more virulent and frequent. Increasing concern about infectious diseases has also brought about developments in statistical and predictive models of viral transmission contributing quantitative indicators of disease and population health worldwide. In this study, many geospatial web services and applications are built for the infectious disease surveillance. It demonstrates that geospatial technologies can support ongoing efforts in disease surveillance and control, public health planning and resource management, identification of high-risk groups, health promotion, prevention of exposures, and reduction in mortalities - with applications that can bridge the policy gap between the global North and South.

Keywords: infectious diseases, surveillance, global health, Geospatial Web Services, web applications.
\end{abstract}

\section{Introduction}

Infectious diseases have shown anti-microbial adaptability and great mobility across countries. Syndromic surveillance for infectious disease requires an ongoing basis, and is a global responsibility of national health authorities. During 2005 and early 2006, highly pathogenic avian influenza (HPAI) H5N1 spread around the globe expanded considerably in both avian host species and 
geographic distribution [1]. The current outbreak of influenza A (H1N1) since April, 2009 made the World Health Organization to increase the alert level to phase 5 out of 6 possible to slow the transmission of the flu pandemic. By May 9, 2009, more than 3400 confirmed H1N1 human cases are reported in 29 countries all over the world. As the expanding of the virus, the risk of an epidemic increases Meanwhile, vaccine production remains unequal and inaccessible to populations most at risk, making immunization strategies difficult to implement at a global scale [2, 3].

With the continuing circulation of virus (such as H1N1 and H5N1), there is an increased possibility of adaptive mutation or genetic reassortment with other circulating influenza viruses causing a global pandemic, especially because effective vaccines may not be available for the first six months or first wave of a pandemic. Thus, pandemic influenza in which an antigenic shift causes an outbreak to which few people are immune by virtue of having no previous exposure, is a growing concern among public health decision makers around the world. While international programs exist to characterize viral isolates and develop vaccines, major challenges to pandemic preparedness remain to be overcome. Syndromic surveillance of flu-like symptoms can be used to trigger more laboratory analysis than is typically done, and thus hasten the public health response [4]. In order to respond to flu outbreaks, it is also necessary to understand the impact of environmental and social factors on the interaction between pathogens and hosts, and understand the patterns of spread through space and time.

Public Health authorities should understand not only the microbiology of infectious agents (its virulence factors), but also the social, demographic, geographic and environmental factors that contribute to its spread. For example, if the Hong Kong influenza pandemic strain of 1968-1969 returned in 2000, according to simulations coupled with air transportation data for 52 cities worldwide, it would spread concurrently to cities in the northern and southern hemispheres [5]. According to the researchers that developed these simulations, these findings highlight the importance of coordinated global surveillance and pandemic planning. Furthermore, this research shows that technology (in this case, air travel) increases the ability of infectious diseases to travel across hemispheres.

More importantly, health deficits, especially in Africa, contribute to underdevelopment, weakened state capacity, leading to population exposure to emerging diseases and weakening global public health protection from environmental and biological threats - amounting to a human rights abridgement [6]. Global health improvement is increasingly an issue of collective international security. Some researchers emphasize the need for the development of policies, procedures, and decision-making in advance of a severe pandemic/epidemic, since there will be insufficient time to prepare after the onset of an epidemic [7]. Improving the performance of health systems has been identified by the WHO as a major global health priority [8]. For example, the creation of national influenza pandemic planning committees and contingency plans in various countries including Canada, US, UK, Switzerland, Australia, 
Japan, France, and Belgium, have been important advancements in pandemic preparedness [9]. In addition, the WHO has published guidelines for National Pandemic Preparedness Planning and Policy.

\section{Geospatial technologies for infectious disease surveillance}

Maps illustrating disease distribution and population health indicators enable authorities to communicate about disease spread, control, and recovery efforts. Geographic Information System (GIS) can be used to illustrate the differences in exposure by thematically rendering syndromic surveillance data along with environmental and population demographic data. GIS can also be used to illustrate differences in access to health care by calculating the average distance traveled and the frequency of cases that are presented in clinics - revealing whether differences in accessibility stem from gaps in service coverage or are biased along class, ethnic, or racial lines [10]. Furthermore, GIS can be used to spatially analyze health systems coverage and identify deficiencies, in the North and South -it already plays a key role in health service planning and resource allocation in Africa [11].

The scalability and analytical capabilities of GIS respond well to the need for local, regional, and international infectious disease surveillance and control. Unlike static maps, the dynamic maps from GIS can be a useful tool for creating global health policy that takes into account regional variations in population health, access to services, disease reservoirs/transmission pathways, global epicenters, and weakest-links in the health chain. Integrating data from the periphery to the center, GIS can eliminate constraints to containing an outbreak and mitigating its effects, protect critical infrastructure, and ensure continuity of government. For example, immunization strategies for seasonal and pandemic influenza in the Global North and South can be developed using location-based data (e.g. human resources and pharmaceutical assets, infrastructure, case tracing, population health, environmental, economic, social and demographic data), as well as through spatial analysis (proximity to epicenters of an outbreak or reservoirs of bird flu). It provides a common operating picture for authorities to effectively respond to health emergencies.

With a growing suite of web-based tools, authorities can take advantage of new opportunities to advance disease surveillance across international borders. Web-based GIS can facilitate inter-disciplinary knowledge (content gathering) and draw together epistemic communities (health, safety, environment), helping policy makers to remove inequalities in basic or universal health care. The implementation of Web-based GIS in support of infectious disease surveillance and control will resolve some of the emerging institutional dilemmas by providing decision-makers across institutions/governments/borders with quick access to closest-to-source data, decision-support applications, automated modeling techniques, and a community-of-practice. Web-based GIS can assist with rapid identification of urgent national risks, including international health emergencies like SARS or pandemic influenza, by seamlessly integrating surveillance data from the periphery to the central government for quick analysis 
and decision-making [12]. The application of Web-GIS to enable evidence-based decision-making would minimize duplication and conflicting actions by diverse agencies in responding to a pandemic.

\section{Developing geospatial web services and application for infectious disease surveillance}

In order to understand the spatial and temporal spread of disease, identify epicenters of outbreaks of emerging or reemerging diseases, track the movement of susceptible populations (high risk groups), determine what the weakest links are, and plan interventions - public health officials need access to population health and syndromic surveillance data that can be mapped over time and space. Quicker and more effective decision-making on emerging and re-emerging infectious diseases could be achieved by integrating standardized and real-time syndromic surveillance data sets seamlessly through web-based mapping applications and services.

Surveillance data required for web-based applications to support decisionmaking in the event of a pandemic, can be obtained only through negotiation between health authorities in different jurisdictions, each with a different approach to disseminating and licensing data in its possession. For public health mapping applications, there needs to be a multi-lateral effort to encourage the use of web-based protocols for communication between web-services accessing standardized public health geospatial data from public health, safety, and environmental agencies (or data custodians) around the world.

Our development of feospatial service framework for infectious disease surveillance is shown in Figure 1. Infectious disease-related data may include: disease surveillance data (e.g. suspected/confirmed cases), health system data (e.g. hospital visits, ICU/bed availability, nurse/doctor availability) socioeconomic or satellite observation data collected by emergency departments, hospitals, clinics, and care facilities. To bring together diverse data and provide decision-makers with value-added information, data mapping and processing functions are provided through geospatial web services. Many standards addressed by ISO/TC211 and Open Geospatial Consortium (OGC) in describing, publishing, visualizing, accessing and manipulating geospatial resources, such as Web Map Service (WMS), Web Coverage Service (WCS), Web Processing Service (WPS) and so on. Many other industrial Web Services developed by ESRI, Google, Yahoo are also very popular in the accessing location-based data. Further more, GeoRSS tagged XML files from GeoRSS services can provide geo-coded infectious disease news. In the Web application design, the services could be mashed up to provide users the desired information for infectious disease surveillance and control. Geospatial Web services and applications that can be applied include many enterprise solutions from Google, Microsoft, ESRI, and open source solutions such as GeoServer, MapServer, OpenLayers.

Several geospatial web services and applications are implemented in our study to facilitate the infectious disease surveillance. The web applications are developed based on OpenLayers. Figure 2 shows an application that integrates 


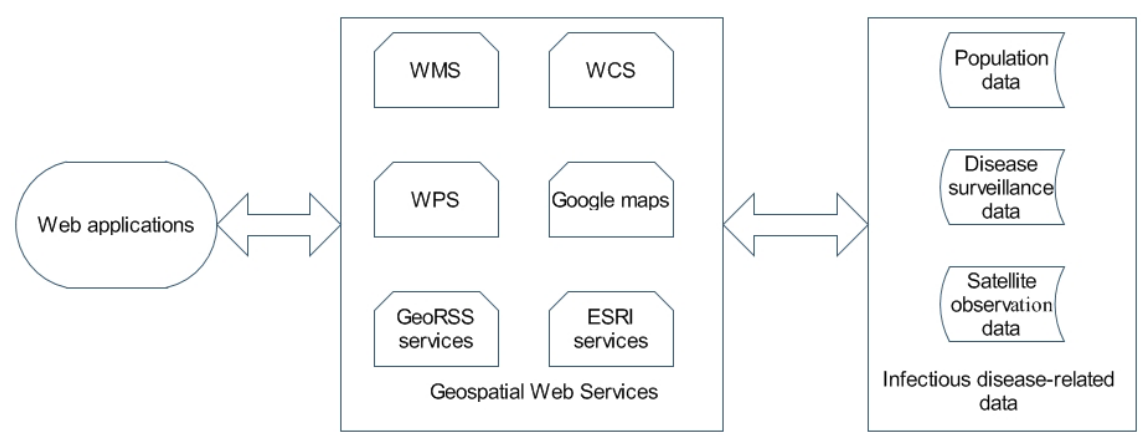

Figure 1: Geospatial service framework for infectious disease surveillance.
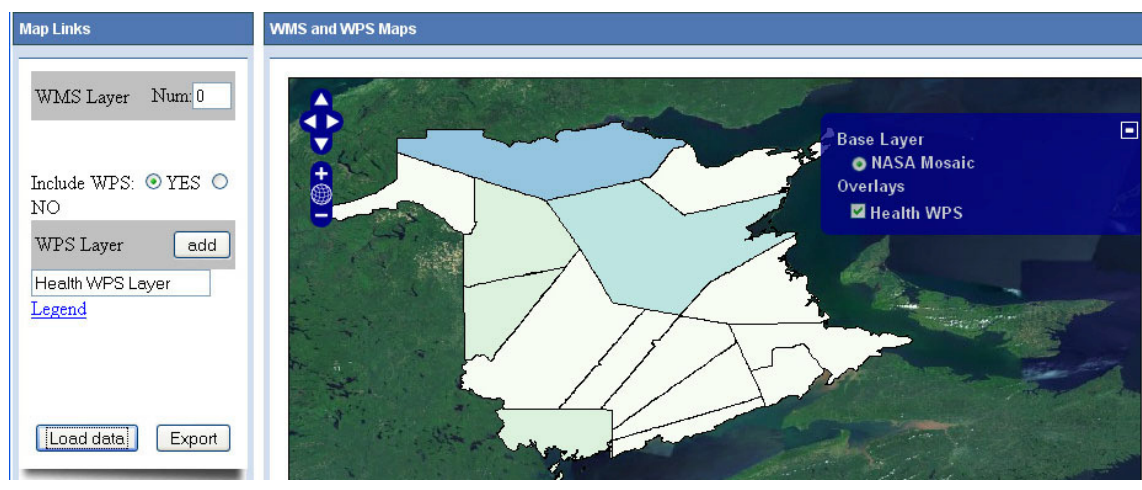

Figure 2: WMS and WPS integration.

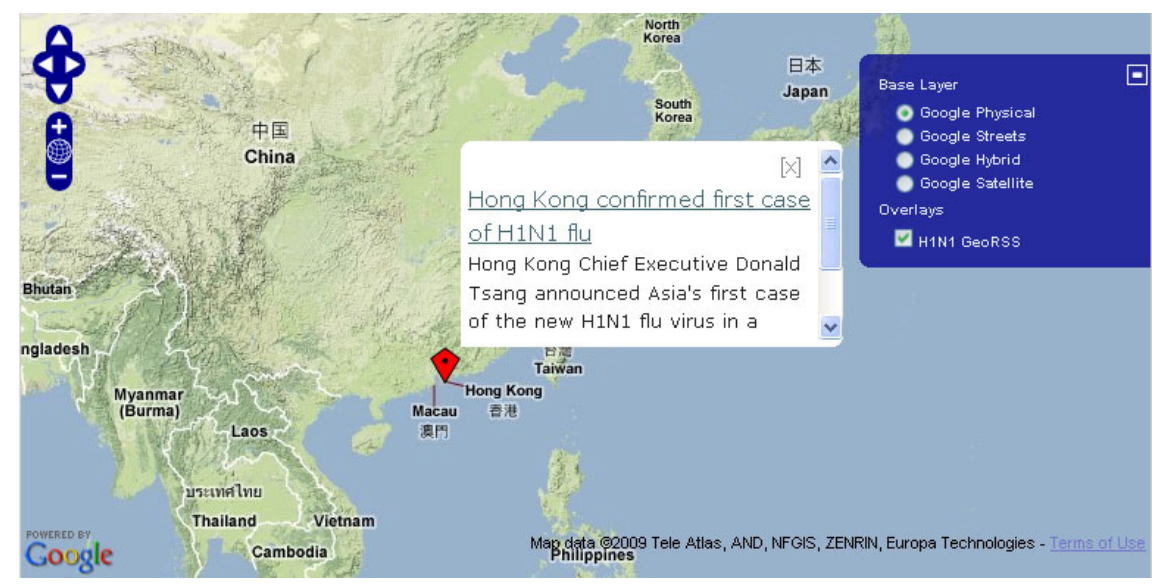

Figure 3: Google maps and GeoRSS service integration. 
WMS and WPS services for integrating distributed data onto a seamless map interface, illustrating health information at various scales, time intervals, and statistical methods. It integrates the NASA WMS with our infectious disease WPS in the example. Figure 3 shows another application that integrates Google maps and our GeoRSS services. In this example, the maps from Google served as a base layer and H1N1 flu GeoRSS information is overlaid.

\section{Conclusions}

The ultimate goal for global and national health policy is to illuminate the road towards implementing a comprehensive national, multi-agency spatio-temporal health information infrastructure functioning proactively in real time [13]. This would provide policy makers and health authorities with maps showing various dimensions of disease, population health, and statistics. As demonstrated above, geospatial web applications are a tool of comparison of the international focus of public health information. Geospatial services and applications can enable map sharing and provide a wider audience with access to automated/analysis functions for infectious disease surveillance \& control. This is important and necessary because we are again facing international health challenges that demand concerted effort.

In conclusion, web-based geospatial application offers a timely solution to a historic challenge - humankind's understanding of ecology and our interconnectedness with each other, the conditions that lead to disease outbreaks and population health vulnerabilities, and how civilization will adapt in order to confront health issues in the $21^{\text {st }}$ Century. If surveillance systems become truly interoperable and offer an abundant variety of map products (illustrating social, environmental and health parameters affecting infection/spread and supporting disease control efforts), the health community will benefit from improved understanding of population health, disease distribution, public health infrastructure, and public health policy in the Global North and South.

Many spatial data custodians already exist - including in the public health community. A continental surveillance system like in the United Kingdom, may provide Canada, the US, Mexico and indeed our foreign partners, with improved pandemic preparedness and outbreak detection capabilities. To build a comprehensive model of population health vulnerabilities, and bridge the northsouth gap when it comes to preventative health policy, will require fundamental upgrading of resources committed, and scaling up of surveillance and disease control applications worldwide. The economy-of-scale is present when epidemiological research and health planning communities utilize a web-GIS system to address inequalities in health care provision, access, and promotion that can be scaled up during outbreak and pandemic response efforts. For a world wide network of data custodians, internet service providers and map application developers, the protocols for sharing information related to infectious disease outbreaks and public health among member states of the United Nations, would need to be further provisioned in a Convention, Resolution or Act. 


\section{References}

[1] Rappole, J.H. \& Hubálek, Z., Birds and influenza H5N1 virus movement to and within North America. Emerging Infectious Diseases, 12, pp. 14861492, 2006.

[2] Knobler, S.L., Mack, A., Mahmoud, A., \& Lemon, S.M., (eds). The Threat of Pandemic Influenza: Are We Ready?, Workshop Summary, Prepared for Forum on Microbial Threats, Board on Global Health, 2005

[3] Kitler, M.E., Gavinio, P., \& Lavanchy, D., Influenza and the work of the World Health Organization. Vaccine, 20, pp. S5-S14, 2002.

[4] Stoto, M.A., Syndromic Surveillance. Issues in Science and Technology, pp. 49-56, 2005.

[5] Grais, R.F., Ellis, J.H., \& Glass, G.E., Assessing the impact of airline travel on the geographic spread of pandemic influenza. European Journal of Epidemiology, 18(11), pp. 1065-1072, 2003.

[6] Janik, L., Boyer, M.A., \& Bobrow, D.B., Progress of Retreat? Global Health Performance and Policy Responses. The annual meetings of the International Studies Association-Northeast, Boston MA, 2006.

[7] Gruber, P.C., Gomersall, C.D., \& Joynt, G.M., Avian influenza (H5N1): implications for intensive care. Intensive Care Med, 32, pp. 823-829, 2006.

[8] WHO, World Health Report 2000: Health systems: Improving Performance. World Health Organization, Geneva, Switzerland.

[9] Theresa, W.S., \& Tam, M.D., Preparing for Influenza Epidemics and Pandemics in the New Millennium. Canadian Journal of Public Health, pp. 293-319, 1999.

[10] Cromley E.K., \& McLafferty S.L., GIS and Public Health, The Guilford Press, pp. 248, 2002.

[11] Tanser, F.C., \& Sueur, D.L., The Application of Geographic Information Systems to important public health problems in Africa International Journal of Health Geographics, 1(4), 2002.

[12] The National Advisory Committee on SARS and Public Health. Learning from SARS: renewal of Public Health in Canada. Ottawa, Health Canada, 2003.

[13] Boulos M.N.K., Towards evidence-based, GIS-driven national spatial health information infrastructure and surveillance services in the United Kingdom, International Journal of Health Geographics, 3(1), 2004. 